\title{
A seroprevalence study to determine the frequency of hantavirus infection in people exposed to wild and pet fancy rats in England
}

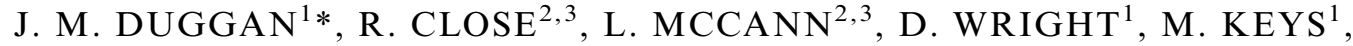 \\ N. MCCARTHY ${ }^{4,5}$, T. MANNES ${ }^{4}$, A. WALSH ${ }^{6}$, A. CHARLETT ${ }^{6}$ AND \\ T. J. G. BROOKS ${ }^{1}$ \\ ${ }^{1}$ Public Health England, Porton Down, Wiltshire, UK \\ ${ }^{2}$ Public Health England, Oxford, UK \\ ${ }^{3}$ European Centre for Disease Prevention and Control, Stockholm, Sweden \\ ${ }^{4}$ Public Health England, Oxford, UK \\ ${ }^{5}$ Warwick Medical School, University of Warwick, Coventry, UK \\ ${ }^{6}$ Public Health England, Colindale, London, UK
}

Received 9 December 2016; Final revision 23 May 2017; Accepted 19 June 2017; first published online 20 July 2017

\section{Summary}

Recent cases of acute kidney injury due to Seoul hantavirus infection from exposure to wild or pet fancy rats suggest this infection is increasing in prevalence in the UK. We conducted a seroprevalence study in England to estimate cumulative exposure in at-risk groups with contact with domesticated and wild rats to assess risk and inform public health advice. From October 2013 to June 2014, 844 individual blood samples were collected. Hantavirus seroprevalence amongst the pet fancy rat owner group was $34.1 \%$ (95\% CI $23.9-45 \cdot 7 \%$ ) compared with $3 \cdot 3 \%$ $(95 \%$ CI $1 \cdot 6-6 \cdot 0)$ in a baseline control group, $2 \cdot 4 \%$ in those with occupational exposure to pet fancy rats (95\% CI $0 \cdot 6-5 \cdot 9)$ and $1 \cdot 7 \%$ with occupational exposure to wild rats (95\% CI $0 \cdot 2-5 \cdot 9)$. Variation in seroprevalence across groups with different exposure suggests that occupational exposure to pet and wild rats carries a very low risk, if any. However incidence of hantavirus infection among pet fancy rat owners/breeders, whether asymptomatic, undiagnosed mild viral illness or more severe disease may be very common and public health advice needs to be targeted to this at-risk group.

Key words: Vector-borne virus, emerging infections, hantavirus, viral haemorrhagic fever, zoonoses.

\section{INTRODUCTION}

Hantaviruses are members of the family Bunyaviridae. There are two types of hantavirus, Old World and New World, which cause different disease aetiology, the severity of illness and target organs largely dependent

\footnotetext{
* Author for correspondence: Dr J. M. Duggan, Rare and Imported Pathogens Laboratory, Public Health England, Porton Down, Wiltshire SP4 0JG, UK.

(Email: Jackie.duggan@phe.gov.uk)
}

on the causative virus. New World hantaviruses cause hantavirus pulmonary syndrome (HPS), a severe cardio-pulmonary disease, while the Old world hantaviruses are present throughout Europe and Asia and are known to cause nephropathia epidemica (NE) and haemorrhagic fever with renal syndrome (HFRS) $[1,2]$. HFRS is an acute disease characterised by sudden onset of fever, lower back pain, varying degrees of haemorrhagic manifestations and renal involvement. However the number of hantavirus infections in humans may be underreported due to asymptomatic 
or mild infection presenting with mild and non-specific symptoms including fever, headache, gastrointestinal symptoms and back pain [3].

Hantaviruses are carried by rodents and insectivores, and different species tend to be associated with a species-specific hantavirus. Animals rarely show signs of disease; they are thought to become infected early in life and may shed virus in their excreta (urine, faeces and saliva) for prolonged periods. In Asia, HFRS is caused mainly by Hantaan virus (HTNV), which is carried by the striped field mouse (Apodemus agrarius), and Seoul virus (SEOV), which is carried by the brown Norway rat (Rattus norvegicus). SEOV was first recognised in Seoul, South Korea, where it was recognised as a milder form of HFRS [4]. From 1979, several outbreaks of HFRS attributed to Seoul virus have been identified in laboratory personnel working with laboratory rats [5-7]. In Europe, HFRS is caused by a number of hantaviruses including Puumala virus (PUUV), carried by the bank vole (Myodes glaeolus), Dobrava virus (DOBV) and Dobrava-like viruses carried by Apodemus flavicollis, Apodemus agrarius and Apodemus ponticus in Europe [1, 2]. HFRS describes a spectrum of disease ranging from sub-clinical to lethal. More severe infections are associated with HTNV and DOBV, whilst milder infections are associated with PUUV and SEOV $[1,2]$. Seoul virus has a worldwide distribution including SE Asia [8,9], the USA $[10,11]$ and Europe $[12,13]$. Phylogenetic analysis has suggested that Seoul virus emerged from Asia into Europe and then into North and South America via trading routes [14]. Transmission of hantaviruses from the rodent host occurs through inhalation of hantavirus-infected, aerosolised excreta [15]. Disease is typically associated with rural workers with close contact with rodents in endemic areas.

Seoul hantaviruses have recently been isolated from wild and pet fancy rats in the UK $[16,17]$, named Humber virus (associated with wild rats) and Cherwell virus (associated with pet rats). The phylogeny of these viruses with Seoul and other hantaviruses has been reported in [16]. Hantavirus infections originating in the UK are rare with a few documented reports of hantavirus seroprevalence [18-29]. The first documented evidence of hantavirus infection in the UK was reported in 1986 from cases of HFRS in laboratory workers working with rats, from which Seoul virus was later isolated from the rats [18]. The virus strain was designated as IR461 and its phylogeny in relation to the other more recently isolated UK hantaviruses is reported in [16].
McKenna et al. conducted a retrospective serosurveillance study on 687 patients presenting with symptoms of HFRS in Northern Ireland and found a $2 \cdot 1 \%$ seropositivity rate using an immunofluorescence assay (IFA) utilising a rat-derived R22VP30 strain of Seoul virus, suggesting that infection originated from exposure to wild rats [19]. Pether and Lloyd found 29 cases of an unspecified hantavirus infection ranging from mild to severe during a serosurveillance study in Somerset conducted as a response to three cases of hantavirus infection in 1991 [20]. A different study was published by Lloyd in 1992 using only HTNV and PUUV in an immunofluorescent assay identifying $21 \cdot 5 \%$ seropositivity for PUUV in UK farmers [21]. This high level of seropositivity has not been replicated in other seroprevalence studies, which have shown an incidence of $4.7 \%$ in a nationwide study of farmers [22] and an incidence of 7.6\% in farmers in Yorkshire [23]. There have been 12 other reported cases of hantavirus infection in the UK before 2012, which have been diagnosed on the basis of serology without virus detection or isolation. From 2012 to 2016, nine cases of hantavirus infection have been confirmed in patients presenting with acute kidney injury (AKI) [16, 17, unpublished data]. All the cases were indigenous, with the patients reporting no travel history and most patients reporting recent exposure to either wild or pet rats. More recently, the virus has also been isolated from wild and pet fancy rats in other European countries, including France [30], The Netherlands [31] and Sweden [32]. A number of Northern European countries, including Belgium, The Netherlands and Germany have reported hantaviruses as being an important cause of AKI with an increasing incidence [33-35]. Given the rising number of cases of AKI due to hantavirus following exposure to pet fancy rats, a seroprevalence study was conducted to assess the risk of acquiring hantavirus from pet rats and from occupational exposure to wild rats to inform public health advice.

\section{METHODS}

\section{Study design and volunteer recruitment}

The study population were those groups who have close contact with domesticated and wild rats in England, with comparison to baseline population. There were four main exposure study groups:

Group 1 (Controls): This group consisted of random blood samples from blood donors purchased through 
Table 1. Sampling numbers and locations for each Study Group

\begin{tabular}{llc}
\hline \hline & & Number of \\
Study group & Event and location & 300 \\
\hline Group 1 & Random stored blood samples purchased from NHSBT & 26 \\
Group 2 (NFRS) & Pet rat owner sampling event 1 - Yorkshire & 32 \\
& Pet rat owner sampling event 2 - Bedfordshire & 21 \\
Group 3 (Veterinary) & Pet rat owner sampling event 3 - Newcastle (North of England) & 170 \\
& British Small Animal Veterinary Association Congress, Birmingham & \\
Group 4 (Farmers) & (nationwide attendance) & 22 \\
& Ross on Wye livestock market, Ross-on-Wye, Herefordshire (Cattle) & 36 \\
& York livestock market, Yorkshire (Pig) & 28 \\
& Sedgemore livestock market, Somerset (Cattle) & 34 \\
Group 4 (Waste water workers) & Ashford livestock Market, Kent (Cattle) & 16 \\
& Waste water treatment centre 1 - West Midlands & 39 \\
& Waste water treatment centre 2 - North West England & 15 \\
Group 4 (Pest control workers) & (Blackburn and Manchester) & 89 \\
& Waste water treatment centre 3 - Yorkshire & 12 \\
& PestTech Conference 2013 (National Pest Technicians Association), & 3 \\
\hline \hline
\end{tabular}

NHSBT, National Health Service Blood and Transplant; NFRS, National Fancy Rat Society.

the National Health Service Blood and Transplant (NHSBT). The results from this group set a baseline percentage cumulative exposure incidence, which may reflect that in the general population.

Group 2: Owners and breeders of pet fancy rats. This group was recruited from members of the National Fancy Rat Society (NFRS) and associated local groups.

Group 3: This group included people working in the pet industry workers who have regular contact with pet fancy rats (small animal veterinarians and pet trade workers).

Group 4: Occupational exposure to wild rat populations. This group comprised volunteers from occupations that are likely to have exposure to wild rats and rat excreta through their occupation. These were farmers, sewer and waste water workers and pest control workers.

A different sampling design was required for each of the study groups based on ease of recruitment. Where possible, a random sample of individuals from the population at risk was obtained from each study group, this is to ensure that the results obtained are generalisable to the populations at risk. Practical aspects of the study logistics presented challenges in executing random sampling designs in the groups and the impact of this is discussed. For all groups except Group 1, the study was publicised using a number of different approaches and a team including recruiters and research nurses attended events shown in Table 1. During the events, individuals were approached by a member of the study team and given written and verbal information on the study.

Given previously reported low prevalence rates of hantavirus seropositivity in the UK, the study aimed to obtain 300 samples for each study group to give a reasonable statistical chance of obtaining positive serology results in any of the at-risk groups. Table 1 shows the number of samples obtained for each study group.

Ethical consent was obtained from the National Research Ethics Committee, reference 13/SW/0117 in July 2013.

\section{Sample collection}

All volunteers recruited to the study were healthy adults $>18$ years and, following written informed consent, gave a blood sample for serological testing. All serum samples were anonymised at the time of collection by giving each a unique number.

\section{Serology procedures}

Blood samples were processed after collection by allowing the samples to clot at room temperature for a 
Table 2. Total number of hantavirus positive sera in each study group

\begin{tabular}{|c|c|c|c|}
\hline Study group & $\begin{array}{l}\text { Total number } \\
\text { of positive } \\
\text { samples }\end{array}$ & $\begin{array}{l}\text { Seroprevalance } \\
(\%)\end{array}$ & $\begin{array}{l}95 \% \text { Confidence } \\
\text { Intervals }\end{array}$ \\
\hline Group 1 (random blood donors) & 10 & $3 \cdot 3$ & $1 \cdot 6-6 \cdot 0$ \\
\hline Group 2 (Pet rat owners) & 27 & $34 \cdot 1$ & $23 \cdot 9-45 \cdot 7$ \\
\hline Group 3 (occupational exposure to pet rats) & 4 & $2 \cdot 4$ & $0 \cdot 6-5 \cdot 9$ \\
\hline Group 4 (occupational exposure to wild rats - Farmers) & 2 & $1 \cdot 7$ & $0 \cdot 2-5 \cdot 9$ \\
\hline Group 4 (Occupational exposure to wild rats - Waste water workers) & 2 & $2 \cdot 9$ & $0 \cdot 3-9 \cdot 9$ \\
\hline Group 4 (Occupational exposure to wild rats - Pest control workers) & 3 & $2 \cdot 8$ & $0 \cdot 6-8 \cdot 0$ \\
\hline Group 4 (total) & 7 & $2 \cdot 4$ & $1 \cdot 0-4 \cdot 8$ \\
\hline
\end{tabular}

minimum of $30 \mathrm{~min}$, followed by centrifugation at 1100 $\boldsymbol{g}$ for $15 \mathrm{~min}$ to separate the serum. Samples were then refrigerated during transport back to the laboratory for further processing and analysis. Serum were analysed using a hantavirus specific IFA (Mosaic 1 slides, EUROIMMUN AG, Lübeck, Germany), containing hantavirus-infected EU14 slides from six hantaviruses (Hantaan, Puumala, Seoul, Saareema, Dobrava, Sin Nombre), described in [36], according to the manufacturer's instructions. The assays are CE-marked and validated according to Directive $98 / 79 / \mathrm{EC}$ on in vitro diagnostic medical devices. The assay has a reported sensitivity of $99 \%$ and specificity of $98 \%$ for IgG [36]. Samples were diluted to $1: 100$ (the starting dilution recommended by the manufacturer) in sample buffer (EUROIMMUN AG) for initial screening and processed using an IF Sprinter automated system (EUROIMMUN AG) with $30 \mu$ diluted sample being added to each reaction field of the mosaic tile. The slides were washed as per the instrument instructions with wash buffer and an anti-human IgG FITC conjugate added automatically. Positive samples were further diluted to $1: 1000$ and $1: 10000$ and processed as before. Processed slides were embedded with mounting medium, cover slipped and evaluated by fluorescence microscopy by an experienced biomedical scientist with no access to the clinical information. Positive reactions were characterised by a fine- to coarse-granular immunofluorescence (IF) in the cytoplasm of infected cells. Intensities of specific IF were compared with those of hantavirus -negative and-positive reference sera and scored as negative, weak, moderate or strong. Samples with at least a weak specific IF at a dilution of $1: 100$ (cut-off) were considered positive. The reciprocal endpoint titre was defined as the highest sample dilution factor for which a weak specific IF was detected. For example, if a serum showed a strong IF at a dilution of $1: 100$, a moderate IF at $1: 1000$ and a negative IF at $1: 10000$, it was assigned a reciprocal endpoint titre of $1: 1000$.

\section{Statistical analysis}

Seroprevalence was calculated for each group. Confidence intervals were calculated around the estimated seroprevalence by using exact binomial confidence intervals for these proportions using Stata version $13 \cdot 1$.

\section{RESULTS}

Between October 2013 and June 2014, we obtained 844 blood samples for analysis, which included 300 random blood donor controls for Study Group 1, 79 samples for Study Group 2, 170 samples for Study Group 3 and 295 samples for Study Group 4. Apart from Study Group 1, the numbers of samples obtained fell short of the 300 samples target for each group. Sampling was random and dependent on accessing and recruiting volunteers, some groups were more difficult to engage in the study than others. To ensure geographical coverage, various events were targeted in different areas of England to recruit volunteers, such as national conferences and meetings (to recruit veterinarians and pest control workers), livestock markets (four in total to cover north, Midlands and south of England to recruit farmers), rat meets and shows organised through the National Fancy Rat Society and water company participation (Yorkshire Water, United Utilities, Severn Trent Water). Table 1 shows the number of samples obtained for each study group and their geographic spread. Table 2 shows the number of positive sera in each group. This is the number of individual sera that reacted with one or more of the six hantaviruses in the IFA. The 
Table 3. Positive samples per study group

\begin{tabular}{|c|c|c|c|c|c|c|}
\hline & HTNV & PUUV & SEOV & SAAV & DOBV & SNV \\
\hline Study Group 1 & $\begin{array}{l}9^{\mathrm{a}}(1 \text { sample } 1: 1000 \\
8 \text { samples } 1: 100)^{\mathrm{b}}\end{array}$ & - & - & - & $1(1: 100)$ & - \\
\hline Study Group 2 & $1(1: 1000)$ & - & $\begin{array}{c}26 \text { (20 samples } 1: 1000,6 \text { samples } \\
1: 10000,1 \text { sample }>1: 10000)\end{array}$ & - & - & - \\
\hline Study Group 3 & $4(1: 100)$ & - & - & - & - & - \\
\hline Study Group 4 (total) & $5(1: 100)$ & $1(1: 1000)$ & - & - & $1(1: 1000)$ & - \\
\hline Group 4 (Farmers) & 1 & 1 & & & & \\
\hline $\begin{array}{l}\text { Group } 4 \text { (Waste water } \\
\text { workers) }\end{array}$ & 2 & & & & & \\
\hline $\begin{array}{l}\text { Group } 4 \text { (Pest control } \\
\text { workers) }\end{array}$ & 2 & & & & 1 & \\
\hline
\end{tabular}

${ }^{a}$ Indicates the hantavirus giving the strongest fluorescence at the end point titre.

${ }^{\mathrm{b}}$ The titre range for the samples is given in parentheses. This is summarised in Table 4 .

Table 4. Summary of end point titres for positive samples

\begin{tabular}{lllll}
\hline \hline & $1: 100$ & $1: 1000$ & $1: 10000$ & $>1: 10000$ \\
\hline Study Group 1 & 9 & 1 (HTNV) & 0 & 0 \\
Study Group 2 & 0 & 20 & 6 & 1 \\
Study Group 3 & 4 & 0 & 0 & 0 \\
Study Group 4 & 5 & 2 & 0 & 0 \\
\hline \hline
\end{tabular}

HTNV, Hantaan virus.

seroprevalence was calculated for each group. The estimated seroprevalence to hantavirus infection in Study Group 2 was $34 \cdot 1 \%$. This means that $34 \cdot 1 \%$ of all samples tested contained hantavirus antibodies, showing previous hantavirus exposure or infection. In comparison, the hantavirus antibody prevalence in the other groups were 3.3\% in Study Group 1, 2.4\% in Study Group 3 and $2 \cdot 4 \%$ in Study Group 4.

Table 3 shows the number of positive samples in each group and the virus that gave the highest IF at the end point titre, whilst Table 4 shows the range of end point titres for each group. Most (26 of 27) of the Study Group 2 positive sera showed broad crossreactivity across the hantavirus group, but most sera gave the strongest reactions against Seoul virus, suggesting that it is likely that those with positive antibody responses were exposed to Seoul virus (Table 3). Twenty-one positive sera with reactivity to hantaviruses were seen in total in the other three study groups, 10 in Group 1, four in Group 3 and seven in Group 4 (Table 3). Two samples (one pest control worker from Study Group 4 and one sample from Study Group 1) showed a stronger positive antibody response for Dobrava, with an estimated seroprevalance of 0.7\% and $0.9 \%$ respectively, whilst one Study Group 4 sample (farmer) tested positive for Puumala antibodies (estimated seroprevalence $0 \cdot 8 \%$ ). Eighteen samples gave a weak positive reaction to Hantaan virus, with 17 of these samples showing no cross-reactivity with any of the other hantaviruses. The samples came from all four study groups with the highest number from study Group 1 with nine samples. These samples were back-titrated to $1: 20$ and $1: 50$ dilutions and gave positive fluorescence at these dilutions (data not shown), and also tested in IFAs against related bunyaviruses at a $1: 100$ dilution of sera (Toscana, Naples, Sicilian and Cyprus sandfly fever viruses, Rift Valley fever virus) using commercially available kits, which all gave a negative reaction (data not shown).

\section{DISCUSSION}

Sampling of volunteers was limited to opportunistic sampling, dependent on recruiting volunteers at specific events being held throughout the country, following liaison with the event co-ordinators. In some cases, it was possible to advertise the study to those attending the events prior to the event, but at many events, the volunteers received verbal and written communication on the study at the time of volunteering. This most probably affected volunteer numbers, especially for some groups that were more difficult to engage in the study than others. Particularly, the pet rat owners in Study Group 2 had concerns about the welfare of their pet rats and the effects of the results of the study on their rats. For study Group 3, the initial intention had been to recruit those working 
in the pet rat industry (breeding and selling rats), as well as small animal veterinarians, but despite much effort from the study team, pet rat industry workers could not be engaged to volunteer for the study.

Study Groups 1, 3 and 4 have a seroprevalence rate of $2 \cdot 4-3 \cdot 3 \%$ for exposure to hantavirus, none of which involved a response predominantly against Seoul virus, meaning that up to $3.3 \%$ of those sampled had positive antibodies to hantaviruses but no evidence for specific immune response against Seoul-like viruses. The results obtained in this study are at odds with previous studies of hantavirus seroprevalence conducted in Northern Ireland [19], which showed that, whilst the seropositivity in the samples obtained was $2 \cdot 1 \%$, the reactivity pattern was almost exclusively to a rat-derived Seoul virus, R22VP30. Other seroprevalence studies in farmers in the UK have shown seropositivity rates of $4 \cdot 8 \%$ [22] and $7 \cdot 6 \%$ [23] with reactions predominantly against Seoul and Hantaan viruses. In this study, whilst reactivity against Seoul virus in non-pet rat owning groups was low, 18 samples from these groups gave a reaction either predominantly or solely to Hantaan virus. It may be inferred from this study that the risk of exposure to hantaviruses in the occupationally exposed groups is not demonstrably higher than the general population. It is also likely that changes to working practices, particularly in farming and increasing use of personal protective equipment has reduced exposure to hantaviruses in the environment, which may explain the lower seroprevalence rates in occupationally exposed groups in this study compared with previous studies.

In contrast, for pet fancy rat owners, the estimated seroprevalence was $34 \cdot 1 \%$ (CI $23 \cdot 9-45 \cdot 7 \%$ ), meaning that a third of those tested had positive antibodies to hantaviruses. The majority of the pet fancy rat owners with a positive antibody response had a strong antibody response with a cross-reactivity pattern suggestive of exposure to a Seoul virus, i.e., the reactivity to Seoul virus was higher than that observed for the other hantaviruses in the panel. Recently, hantavirus was found by polymerase chain reaction (PCR) in the urine of a UK patient with AKI, which on subsequent sequencing was shown to be Cherwell virus, a variant of Seoul virus found in pet rats in the UK (Dr Emma Aarons, Dr Lisa Jameson, personal communication). This is the first time that the virus has been demonstrated directly in a human clinical sample in the UK. The patient had recently acquired pet fancy rats from a local breeding colony. Given previous reports of infection in pet fancy rat owners with Seoul virus, together with PCR evidence of Cherwell variant Seoul virus in sanguinised pet fancy rats [16], we conclude that the virus has been widespread in the specialised pet fancy rat community in England. This study provides evidence for extensive exposure to hantavirus across the specialist rat owning and breeding population in the UK, especially as those recruited to the study had come from areas throughout England travelling to the rat shows/meets where recruitment took place (data not shown). In combination with the increasing recognition of clinical cases in this group and their family members, this is strong evidence for Cherwell variant Seoul hantavirus endemicity, at least in this segment of the UK fancy rat population. The high percentage of antibody positive owners suggests that this virus is widely present in pet fancy rats and presents a significant risk of infection to owners and breeders of this group of pet fancy rats. Given the strong evidence for endemicity among pet fancy rat owners and breeders and the lack of evidence for risk to veterinarians the risk to those breeding and owning non-specialist pet fancy rats is an important question. It is estimated that $0 \cdot 1 \%$ of UK households owned pet fancy rats in 2014 [37], suggesting there is a potential for an elevated risk of hantavirus infection, with a range of presentations from asymptomatic through to HFRS, to a substantial population being exposed. Public health advice has been written aimed at pet fancy rat owners to limit exposure to fomites and published on the PHE website [38], as well as being distributed to the specialist pet fancy rat owning community through the National Fancy Rat Society. More research is required to determine the risk of infection from other domesticated rats in the UK, such as rats bred for the commercial pet rat trade and rats bred as feeder rats (fed to reptiles).

A number of samples gave an antibody pattern of low titre antibodies against other hantaviruses (Tables 3 and 4). Most of these samples (19/22) gave a positive reactivity pattern against Hantaan virus, a virus that causes severe HFRS and is not known to exist outside central and eastern Asia. Previous seroprevalance studies in the UK have shown seropositivity predominantly against Seoul virus, suggesting exposure to rats and cross-reactivity between Seoul and Hantaan is common given that both viruses belong to the Murinae line of hantaviruses, as opposed to viruses such as Puumala and Tula, which are associated with Arvicolinae. Whilst it is highly unlikely that HTNV is found in the UK, especially given that the rodent host (A. agrarius) is not 
found here, an alternative theory to explain the number of positive reactivity patterns to HTNV is this, may be indicative of an, as yet unidentified, hantavirus that may be present in the UK. A number of other mammalian species, including insectivores such as shrews and moles, and insectivorous bats have been found to harbour hantaviruses [39], whilst a novel hantavirus, Tatenale virus, has previously been found in field voles in the UK [40, 41], suggesting the possibility that other rodent species in the UK harbour hantaviruses. Three reactions to other hantaviruses were observed, one to PUUV in a farmer, one to DOBV in a pest control worker and one to DOBV in a control blood donor serum. These reactions may indicate that PUUV and DOBV are present in the UK, but at low volumes given the lack of evidence of these viruses in UK rodents and the effect of ecology and the environment as discussed in [42] or may represent cross-reactivity to other indigenous hantaviruses, such as TATV, through exposure to rodents in the environment.

This study has highlighted the risk of hantavirus infection transmitted from pet fancy rats in England. There may be a risk of hantavirus infection in exposure groups not included in this study. In addition, investigations into the carriage of hantaviruses in indigenous wild rodent populations would enhance our knowledge of the ecology and epidemiology of this group of viruses in the UK. It is important to raise awareness amongst clinicians of the risk of hantavirus infection in those with pet fancy rat contact and the possible risk for non-fancy rat contact such as commercially sourced pet rats and feeder rats. In addition, the risk of hantavirus infection from exposure to wild rats remains a real, if much lower risk to those with environmental exposure to wild rats.

\section{ACKNOWLEDGEMENTS}

The authors would like to thank Dr Lisa Ottowell (nee Jameson) and Dr Emma Aarons for their technical help and advice, Sophie May, Renee Peterson, Katie Russell, Linda Parr, Stella Botchway, Sophie Newitt, Jo Jeffries and Sarah Foulks for volunteering to recruit study participants, the National Fancy Rat Society for their help in engaging with rat owners and to arrange sampling sessions at NFRS meetings and shows, and the following organisations for allowing us to access their premises and conferences to recruit volunteers: British Small Animal Veterinary Association, National Pest Technicians Association and PestTech, Sedgemoor
Livestock Market, Ross-on-Wye Livestock Market, York Livestock Market, Ashford Livestock Market, Yorkshire Water, United Utilities, Severn Trent Water. This research received no specific grant from any funding agency, commercial or not-for-profit sectors.

\section{DECLARATION OF INTEREST}

None.

\section{ETHICAL STANDARDS}

The authors assert that all procedures contributing to this work comply with the ethical standards of the relevant national and institutional committees on human experimentation and with the Helsinki Declaration of 1975, as revised in 2008 .

\section{REFERENCES}

1. Jonsson CB, Figueiredo LT, Vapalahti O. A global perspective on hantavirus ecology, epidemiology and disease. Clinical Microbiology Review 2010; 23: 412-441.

2. Clement J, Maes P, Van Ranst M. Hemorrhagic fever with renal syndrome in the new, and hantavirus pulmonary syndrome in the old world: paradi(se)gm lost or regained? Virus Research 2014; 187: 55-58.

3. Bi Z, Formenty PBH, Roth CE. Hantavirus infection: a review and global update. Journal of Infection in Developing Countries 2008; 2: 3-23.

4. Lee H, Baek LJ, Johnson KM. Isolation of Hantaan virus, the etiological agent of Korean hemorrhagic fever, from wild urban rats. Journal of Infectious Disease 1982; 146: 638-644.

5. Umenai T, et al. Korean haemorrhagic fever in staff in an animal laboratory. Lancet 1979; 1: 1314-1316.

6. Liu RH, Chen HX. The risk and prevention of hemorrhagic fever with renal syndrome transmitted by laboratory rats. Chinese Journal of VectorBilogyo Control. 1991; 2(Suppl.): 250-254.

7. Lloyd G, et al. HFRS outbreak associated with laboratory rats in UK. Lancet 1984; 1: 1175-1176.

8. Zhang YZ, et al. Seoul virus and hantavirus disease, Shenyang, People's Republic of China. Emering Infectious Disease 2009; 15: 200-206.

9. Arikawa J, et al. Epidemiological studies of hemorrhagic fever with renal syndrome related virus infection among urban rats in two islands in Tokyo Bay, Japan. Acta Virology 1985; 29: 66-72.

10. LeDuc JW, Smith GA, Johnson KM. Hantaan-like viruses from domestic rats captured in the United States. American Journal of Tropical Medicine and Hygiene 1984; 33: 992-998.

11. Korch GW, et al. Serological evidence of hantaviral infections within small mammal communities in Baltimore, Maryland: spacial and temporal patterns 
and host range. American Journal of Tropical Medicine and Hygiene 1989; 41: 230-240.

12. Heymann $\mathbf{P}$, et al. Seoul hantavirus in Europe: first demonstration of the virus genome in wild Rattus norvegicus captured in France. European Journal of Clinical Microbiology and Infectious Disease 1984; 23: 711-717.

13. Heymann $\mathbf{P}$, et al. Serological and genetic analysis for the presence of Seoul hantavirus in Rattus norvegicus in Flanders, Belgium. Scandinavian Journal of Infectious Disease 1989; 41: 51-56.

14. Lin XD, et al. Migration of Norway rats resulted in the worldwide distribution of Seoul hantavirus today. Journal of Virology 2012; 86: 972-981.

15. McCaughy C, Hart CA. Hantaviruses. Journal of Medical Microbiology 2000; 49: 587-599.

16. Jameson LJ, et al. The continued emergence of hantaviruses: isolation of a seoul virus implicated in human disease, United Kingdom, October 2012. Eurosurveillance 2013; 18: 1-4.

17. Jameson LJ, et al. Pet rats as a source of hantavirus in England and Wales, 2013. Eurosurveillance 2013; 18: 20415-20418.

18. Lloyd G, Jones N. Infection of laboratory workers with hantavirus acquired from immunocytomas propagated in laboratory rats. Journal of Infection 1986; 12: 117-125.

19. McKenna P, et al. Serological evidence of Hantavirus disease in Northern Ireland. Journal of Medical Virology 1994; 43: 33-38.

20. Pether JV, Lloyd G. The clinical spectrum of human hantavirus infection in Somerset, UK. Epidemiology and Infection 1993; 111: 171-175.

21. Lloyd G. Hantavirus. In: Peter M-C, ed. Current Topics in Clinical Virology. Cambridge, UK: Cambridge University Press, 1991: pp. 181-204.

22. Coleman TJ. The public health laboratory service (PHLS) and its role in the control of zoonotic disease. Acta Tropica 2000; 76: 71-75.

23. Jameson LJ, et al. Prevalence of antibodies against hantaviruses in serum and saliva of adults living or working on farms in Yorkshire, United Kingdom. Viruses 2014; 6: $524-534$.

24. Walker E, et al. A Scottish case of nephropathy due to Hantaan virus infection. Journal of Infection 1985; 11: $57-58$.

25. Kudesia G, et al. Dual infection with leptospira and hantavirus. Lancet 1988; 1: 1397.

26. Watson AR, et al. Playing in a scrapyard and acute renal failure. Lancet 1997; 349: 1446.
27. Pether JV, et al. Acute hantavirus infection. Lancet 1991; 338(8773): 1025.

28. Phillips MJ, et al. Further UK case of acute hantavirus infection. Lancet 1991; 14: 1530-1531.

29. Rice P, et al. Acute hantavirus infection. Journal of Infection 1993; 27: 342-4.

30. Dupinay T, et al. Detection and genetic characterisation of Seoul virus from commensal brown rats in France. Journal of Virology 2014; 11: 32-40.

31. Verner-Carlsson J, et al. First evidence of Seoul virus in the wild rat population in The Netherlands. Infection Ecology and Epidemiology 2015; 5: 27215-27219.

32. Lundkvist A, et al. Pet rat harbouring Seoul hantavirus in Sweden, June 2013. Eurosurveillance 2013; 18: 20521 20524.

33. Clement $\mathbf{J}$, et al. Relating increasing hantavirus incidences to climate change: the mast connection. International Journal of Health Geography 2009; 8: 1-11.

34. Groen $\mathbf{J}$, et al. Hantavirus infections in The Netherlands: epidemiology and disease. Epidemiology and Infection 1995; 114: 373-383.

35. Krautkrämer E, et al. Recent outbreaks of hantavirus disease in Germany and the United States. Kidney International 2012; 82: 1243-1245.

36. Lederer $\mathbf{S}$, et al. Indirect immunofluorescence assay for the simultaneous detection of antibodies against clinically important old and new world hantaviruses. PLoS Neglected Tropical Diseases 2013; 74: e2157.

37. Pet Food Manufacturer's Association. Pet population. 2014 (http://www.pfma.org.uk/pet-population). Accessed 10 March 2017.

38. Public Health England. Pet rats, mice, hamsters: reducing the risk of infection (https://www.gov.uk/government/ publications/pet-rats-mice-hamsters-reducing-the-risk-ofinfection). Accessed 10 March 2017.

39. Bennett SN, et al. Reconstructing the evolutionary origins and phylogeography of hantaviruses. Trends in Microbiology 2014; 22: 473-482.

40. Pounder KC, et al. Novel hantavirus in field vole, United Kingdom (letter). Emerging Infectious Diseases 2013; 19: 673-675.

41. Thomason AG, et al. An endemic hantavirus in field voles in northern England. Emerging Infectious Diseases 2017; 23: 1033-1035.

42. Bennett E, et al. Environmental and ecological potential for enzootic cycles of Puumala hantavirus in Great Britain. Epidemiology and Infection 2010; 138: 91-98. 